\title{
Differentiation of breast tuberculosis and breast cancer using diffusion-weighted, T2-weighted and dynamic contrast-enhanced magnetic resonance imaging
}

\begin{tabular}{|c|c|}
\hline \multicolumn{2}{|c|}{$\begin{array}{l}\text { Authors: } \\
\text { Dibuseng P. Ramaema }{ }^{1} \text { (D) } \\
\text { Richard J. Hift }{ }^{2} \text { (D) }\end{array}$} \\
\hline \multicolumn{2}{|c|}{$\begin{array}{l}\text { Affiliations: } \\
{ }^{1} \text { Division of Radiation } \\
\text { Medicine, Nelson R Mandela } \\
\text { School of Medicine, } \\
\text { University of KwaZulu-Natal, } \\
\text { South Africa }\end{array}$} \\
\hline \multicolumn{2}{|c|}{$\begin{array}{l}{ }^{2} \text { Division of Medicine, Nelson } \\
\text { R Mandela School of } \\
\text { Medicine, University of } \\
\text { KwaZulu-Natal, South Africa }\end{array}$} \\
\hline \multicolumn{2}{|c|}{$\begin{array}{l}\text { Corresponding author: } \\
\text { Dibuseng Ramaema, } \\
\text { ramaemad@ukzn.ac.za }\end{array}$} \\
\hline \multicolumn{2}{|c|}{$\begin{array}{l}\text { Dates: } \\
\text { Received: } 16 \text { June } 2018 \\
\text { Accepted: } 06 \text { Sept. } 2018 \\
\text { Published: } 25 \text { Oct. } 2018\end{array}$} \\
\hline \multicolumn{2}{|c|}{$\begin{array}{l}\text { How to cite this article: } \\
\text { Ramaema DP, Hift RJ. } \\
\text { Differentiation of breast } \\
\text { tuberculosis and breast } \\
\text { cancer using diffusion- } \\
\text { weighted, T2-weighted and } \\
\text { dynamic contrast-enhanced } \\
\text { magnetic resonance imaging. } \\
\text { S Afr J Rad. 2018; 22(2), } \\
\text { a1377. https://doi.org/ } \\
\text { 10.4102/sajr.v22i2.1377 }\end{array}$} \\
\hline \multicolumn{2}{|c|}{$\begin{array}{l}\text { Copyright: } \\
\text { (C) 2018. The Authors } \\
\text { Licensee: AOSIS. This } \\
\text { is licensed under the } \\
\text { Creative Commons } \\
\text { Attribution License. }\end{array}$} \\
\hline \multicolumn{2}{|l|}{ Read online: } \\
\hline 口iti: & $\begin{array}{l}\text { Scan this QR } \\
\text { code with your } \\
\text { smart phone or } \\
\text { mobile device } \\
\text { to read online. }\end{array}$ \\
\hline
\end{tabular}

Background: The use of multi-parametric magnetic resonance imaging (MRI) in the evaluation of breast tuberculosis (BTB).

Objectives: To evaluate the value of diffusion-weighted imaging (DWI), T2-weighted (T2W) and dynamic contrast-enhanced magnetic resonance imaging (DCE-MRI) in differentiating breast cancer (BCA) from BTB.

Method: We retrospectively studied images of 17 patients with $B C A$ who had undergone preoperative MRI and 6 patients with pathologically proven BTB who underwent DCE-MRI during January 2014 to January 2015.

Results: All patients were female, with the age range of BTB patients being 23-43 years and the BCA patients being 31-74 years. Breast cancer patients had a statistically significant lower mean apparent diffusion coefficient $(\mathrm{ADC})$ value $(1072.10 \pm 365.14)$, compared to the BTB group $(1690.77 \pm 624.05, p=0.006)$. The mean T2-weighted signal intensity (T2SI) was lower for the BCA group $(521.56 \pm 233.73)$ than the BTB group $(787.74 \pm 196.04, p=0.020)$. An ADC mean cut-off value of 1558.79 yielded $66 \%$ sensitivity and $94 \%$ specificity, whilst the T2SI cut-off value of 790.20 yielded $83 \%$ sensitivity and $83 \%$ specificity for differentiating between BTB and BCA. The homogeneous internal enhancement for focal mass was seen in BCA patients only.

Conclusion: Multi-parametric MRI incorporating the DWI, T2W and DCE-MRI may be a useful tool to differentiate BCA from BTB.

\section{Introduction}

Although breast cancer (BCA) is the most prevalent malignancy amongst South African women, accounting for $20.62 \%,{ }^{1}$ the prevalence of breast tuberculosis (BTB) is by contrast much lower, with reported ranges between $0.3 \%$ and $0.4 \%{ }^{2,3}$ Breast tuberculosis is a manifestation of extrapulmonary tuberculosis (EPTB), a condition which has become considerably more commonly encountered as a consequence of the human immunodeficiency virus (HIV) pandemic; 4,5 yet, studies and guidelines on the management of EPTB frequently do not mention the breast as a potential site for tuberculosis. ${ }^{6,7,8}$ Given the infrequency with which BTB is diagnosed, little research has been performed on the disease. Breast tuberculosis and BCA may present clinically in a very similar fashion leading to potential misdiagnosis ${ }^{9,10}$; given that an accurate diagnosis is essential if the patient is to receive correct and timely treatment, this may be problematic. ${ }^{8}$

Conventional imaging with mammography and ultrasound has limited ability to differentiate between BTB and BCA. ${ }^{11}$ It is therefore appropriate to investigate the use of newer and more complex imaging modalities in the differentiation of BCA and BTB. Studies that have used magnetic resonance imaging (MRI) to differentiate EPTB from malignant lesions are those which focused on other body parts such as the central nervous system (CNS) ${ }^{12,13,14}$ and the musculoskeletal system. ${ }^{15,16}$ Peng et al. used the diffusion-weighted imaging (DWI) and magnetic resonance spectroscopy (MRS) to differentiate intracranial tuberculomas from high-grade gliomas and found significant differences. The diagnostic accuracy was higher when using the minimum apparent diffusion coefficient (ADC) value from the DWI than the maximum MRS ratios of Cho/ $\mathrm{Cr}, \mathrm{Cho} / \mathrm{NAA}$ and Cho/Cho. ${ }^{13}$ Magnetic resonance imaging has also been utilised in pulmonary tuberculosis (PTB) to differentiate between tuberculous and malignant nodules. ${ }^{17,18,19,20}$ Only one 
study was found in the literature which aimed to differentiate BTB from BCA with the use of MRS in four BTB patients with no comparison group. ${ }^{21}$ The authors suggested that the absence of choline peak and the presence of a strong lipid peak favoured BTB rather than BCA. ${ }^{21}$

There are multiple studies that utilise DWI and ADC to distinguish malignant from benign breast disease. .2, 23,24,25,26,27 $^{2}$ Diffusion-weighted imaging measures the microscopic movement of water molecules in biological tissues, with the pathologic processes altering their mobility; the detection of these changes aids in lesion characterisation. ${ }^{28}$ The ADC is derived from DWI sequences. Apparent diffusion coefficient values, measured in $\mathrm{mm}^{2} / \mathrm{s}$, are calculated within a given area. This reflects water restriction. Tissues with high ADC values, because of increased diffusion, display a brighter signal, whereas those with lower ADC values appear darker, because of restricted diffusion. ${ }^{29}$ Most malignant lesions display lower ADC values when compared to benign or inflammatory lesions. ${ }^{26}$ Rong-Feng $\mathrm{Qu}$ et al. recently conducted a meta-analysis of the differential diagnosis of benign and malignant breast tumours. The authors found that the ADC values of normal breast tissues were higher than those of benign tissues, and that the values of benign lesions were higher than those of malignant tumours. ${ }^{23}$

Breast dynamic contrast-enhanced magnetic resonance imaging (DCE-MRI) involves administration of a magnetic compatible intravenous (IV) contrast agent in order to detect and characterise lesions. ${ }^{30}$ Further advantage is the ability to evaluate the breast lesion enhancement parameters and kinetic curves. It has been demonstrated that during the wash-in rate, malignant lesions demonstrated higher maximum enhancement when compared to benign lesions. ${ }^{31}$ Dynamic contrast-enhanced MRI kinetic characteristics have been shown to correlate with tumour neovascularity in which more vascular tumours demonstrate strong enhancement. ${ }^{32}$

The T2-weighted (T2W) MRI uses the tissue transverse relaxation times to generate the signal. ${ }^{33}$ The displayed T2 signal intensity (T2SI) is used in the characterisation of tissues and lesions. ${ }^{33}$ Studies that used the T2SI in the discrimination of benign and malignant breast lesions mainly analysed the morphologic T2SI appearance rather than the quantitative values. ${ }^{34,35}$ The majority of BCAs appear hypointense on T2W, compared to most benign lesions that appear hyperintense. ${ }^{36}$

The purpose of our study was to evaluate the use of the DWI parameter of ADC, the DCE-MRI enhancement morphologic characteristics and the T2W parameter of T2SI value to differentiate BCA from BTB.

\section{Patients and methods}

We retrospectively identified 24 patients with histologically proven BCA (including ductal carcinoma in situ [DCIS]) who underwent DWI, T2W and DCE-MRI during January 2014 to December 2014. Seven patients did not have a full set of images on the picture archiving and communication system
(PACS) and were excluded from the study, which resulted in the final total of 17 patients.

For the BTB group, we included six prospectively and consecutively identified patients with proven BTB who also underwent the DWI, T2W and DCE-MRI. Although five patients had conclusive histology from either the breast or the axillary lymph nodes, one patient had inconclusive histology from breast and axilla, but had proven concurrent PTB, for which she was receiving anti-tuberculous therapy (ATT). Her clinical and radiological features were compatible with BTB, and both the breast and the axillary nodes responded satisfactorily to ATT. All BTB patients had undergone screening investigations at the referring breast clinic, including ultrasonography, with or without mammograms, depending on their age. Following the histological confirmation of BTB, the patients were invited for a baseline MRI scan within 2 weeks of confirmation of the diagnosis. Scans were not performed within 2 weeks of a biopsy in order to avoid the presence of haematoma and/or inflammation confounding our results.

\section{Magnetic resonance imaging image acquisition}

Magnetic resonance imaging images were retrospectively analysed following retrieval from the hospital's PACS. Magnetic resonance imaging was performed on a 1.5 Telsa machine (Siemens, Erlangen, Germany) using a dedicated breast coil. The patients were scanned in the prone position. A power injection of $20 \mathrm{~mL}$ intravenous (IV) Magnevist ${ }^{\circledR}$ (Gadopentetate Dimeglumine, Bayer) Standard $469 \mathrm{mg} / \mathrm{mL}$ $(0.5 \mathrm{mmol} / \mathrm{mL})$ at a dosage of $0.1 \mathrm{mmol} / \mathrm{kg}$ was administered to all the patients. The IV contrast rate was $3 \mathrm{~mL} / \mathrm{s}$ followed by a $20 \mathrm{~mL}$ saline flush administered as a bolus. The T1 dynamic phase scan time was 6 min $41 \mathrm{~s}$, during which five dynamic sequences were obtained in the axial position at various time points. The DWI images with the ADC map were also acquired during the same scan. The technical parameters are reported in Table 1.

\section{Magnetic resonance imaging image analysis}

All the images were read by one of the authors who is a radiologist with more than 10 years' experience in breast radiology. The following qualitative parameters were recorded:

- The BCA pathological subtype from the pathology records.

- The DCE-MRI parameters were classified using the Breast Imaging Reporting and Data System (BI-RADS) descriptors ${ }^{37}$ and included the following:

- The enhancement pattern: Either mass-like enhancement (MASS) or non-mass enhancement (NME).

- Non-mass enhancement distribution pattern: Focal, linear, segmental, regional, multiple regions or diffuse.

- Non-mass enhancement internal enhancement: Homogeneous, heterogeneous, clumped and clustered ring.

- MASS internal enhancement: Homogeneous, heterogeneous, rim enhancement and dark internal septations. 
TABLE 1: Local dynamic contrast-enhanced breast magnetic resonance imaging protocol technical parameters.

\begin{tabular}{|c|c|c|c|c|c|c|c|c|}
\hline MRI sequence & Acquisition plane & TR (ms) & $\mathrm{TE}(\mathrm{ms})$ & $\begin{array}{l}\text { Inversion time } \\
\text { (ms) }\end{array}$ & Matrix size & FoV $(\mathrm{mm})$ & $\begin{array}{l}\text { Slice thickness } \\
(\mathrm{mm})\end{array}$ & Voxel size $(\mathrm{mm})$ \\
\hline Localiser & sagittal & 7.6 & 3.53 & - & $384 \times 512$ & 400 & 6 & $2.1 \times 1.6 \times 6.0$ \\
\hline T1 pre-contrast GE 3D & axial & 8.6 & 4.70 & - & $299 \times 384$ & 320 & 1 & $1.0 \times 0.7 \times 1.0$ \\
\hline $\begin{array}{l}\text { T1 GE 3D dynamic sequences } \\
\text { (1 pre-contrast and } 5 \text { post-contrast) }\end{array}$ & axial & 9.1 & 4.76 & - & $299 \times 284$ & 340 & 1.5 & $1.1 \times 0.9 \times 1.5$ \\
\hline T1 3D Dixon & axial & 7.20 & $\begin{array}{l}\text { First } 2.38 ; \\
\text { second } 4.76\end{array}$ & - & $320 \times 320$ & 340 & 1.8 & $1.1 \times 1.1 \times 1.8$ \\
\hline T1 fat saturated & axial & 680 & 10 & - & $224 \times 320$ & 320 & 4 & $1.4 \times 1.0 \times 4.0$ \\
\hline T2 STIR & axial & 5600 & 59.0 & 170 & $314 \times 320$ & 340 & 4 & $1.1 \times 1.1 \times 4.0$ \\
\hline T2 TSE & axial & 6100 & 111 & - & $384 \times 512$ & 320 & 4 & $1.7 \times 1.3 \times 4.0$ \\
\hline DWI between values 0 and $800 \mathrm{~s} / \mathrm{mm}^{2}$ & axial & 9200 & 86 & 180 & $150 \times 192$ & 380 & 4 & $2.0 \times 2.0 \times 4.0$ \\
\hline
\end{tabular}

MRI, magnetic resonance imaging; TR, repetition time; TE, echo time; FoV, field of view; DWI, diffusion-weighted imaging; TSE, turbo spin echo; GE, gradient echo; STIR, short tau inversion recovery.

- The presence or absence of axillary nodes was added to the above descriptors.

We further recorded the ADC values $\left(\times 10^{-6} \mathrm{~mm}^{2} / \mathrm{s}\right)$ derived from the DWI images, and T2SI value derived from the T2W. For the quantitative parameters, the region of interest (ROI) was drawn on three contiguous axial images around the lesion circumference and the average recorded. All images were analysed on the dedicated Syngo-via (Siemens, Erlangen, Germany) reading platform.

\section{Statistical analysis}

Data were entered into an Excel spreadsheet (Microsoft Excel 2013. Redmond, WA) and analysed using the STATA software package (StataCorp. 2015: Stata Statistical Software, Release 14. College Station, TX). Differences in ADC value and T2SI between the two groups were assessed with a two-tailed unpaired $t$-test. Differences in ADC value and T2SI with enhancement pattern matching were assessed using analysis of variance (ANOVA). The results were considered significant where $p<0.05$.

\section{Ethical consideration}

Ethical clearance was obtained from the institution's Biomedical Research Ethics Committee (BREC) (Reference number BF213/13).

\section{Results}

\section{Patient characteristics}

All the patients were female. The ages of the BTB patients ranged from 23 to 43 years, and for those with BCA, 31-74 years. Three BTB patients were HIV positive, one HIV negative and two not tested. The BCA subtypes included 13 cases of invasive ductal cancer (IDC) $(76.5 \%) ; 3$ cases of DCIS $(17.6 \%)$, of whom one had high-grade DCIS; and 1 case $(5.9 \%)$ of invasive lobular cancer (ILC).

\section{Radiologic findings}

The MRI morphological enhancement characteristics of the BCA patients are shown in Figure 1 and those of BTB in Figure 2.

\section{Mass versus non-mass enhancement pattern}

In the patients with BTB, 3/6 (50\%) had mass-like enhancement, and $3 / 6$ (50\%) had NME patterns.

In the patients with BCA, 11/17 (65\%) had mass-like enhancement, and 6/17 (35\%) had NME patterns.

\section{Enhancement patterns}

Amongst the BTB patients, 3 (50\%) had clumped diffuse NME, $2(33 \%)$ showed focal mass rim and $1(17 \%)$ demonstrated multiple regions mass rim. Axillary nodes in BTB patients demonstrated rim enhancement (Figure 2).

Of the BCA patients, $5(29 \%)$ had heterogeneous enhancement, 7 (41\%) had homogeneous enhancement, $4(24 \%)$ had clumped enhancement and $1(6 \%)$ had clustered ring enhancement (Figure 1).

\section{Apparent diffusion coefficient and T2-weighted signal intensity values}

The representative images demonstrating data extraction for ADC values and T2SI measurements of the BCA and BTB patients are depicted in Figure 3.

We found significant differences in both ADC and mean T2SI values between the two groups. The mean ADC for BTB was $1690.8 \pm 624.1$, and for BCA, it was 1072.1 $\pm 365.1(p=0.006)$. The mean T2SI for BTB was $787.7 \pm 196.0$, and for BCA, it was $521.6 \pm 233.7(p=0.020)$ (Table 2$)$.

With regard to the ADC values, receiver operating characteristic (ROC) curve analysis showed a sensitivity of $66.7 \%$ (CI $22.3 \%-$ 95.7\%) and specificity of $94.1 \%$ (CI 71.3\% - 99.9\%) for a diagnosis of BTB at a cut-off value of $152.2 \times 10^{6} \mathrm{~mm}^{2} / \mathrm{s}$, with an area under the curve (AUC) of 0.81 (CI $0.60 \%-0.94 \%$ ) (Figure 4 ).

The corresponding values for T2SI were $83.3 \%$ specificity (CI 35.9\% - 99.6\%) and 82.4\% sensitivity (CI 56.6\% - 96.2\%) at a cut-off value of 670.6 , with an AUC of 0.77 (CI $0.54 \%-0.91 \%$ ) (Figure 5).

\section{Breast cancer versus breast tuberculosis with matched patterns}

These differences persisted when the two groups were matched for the patterns of mass-like enhancement (MASS) 

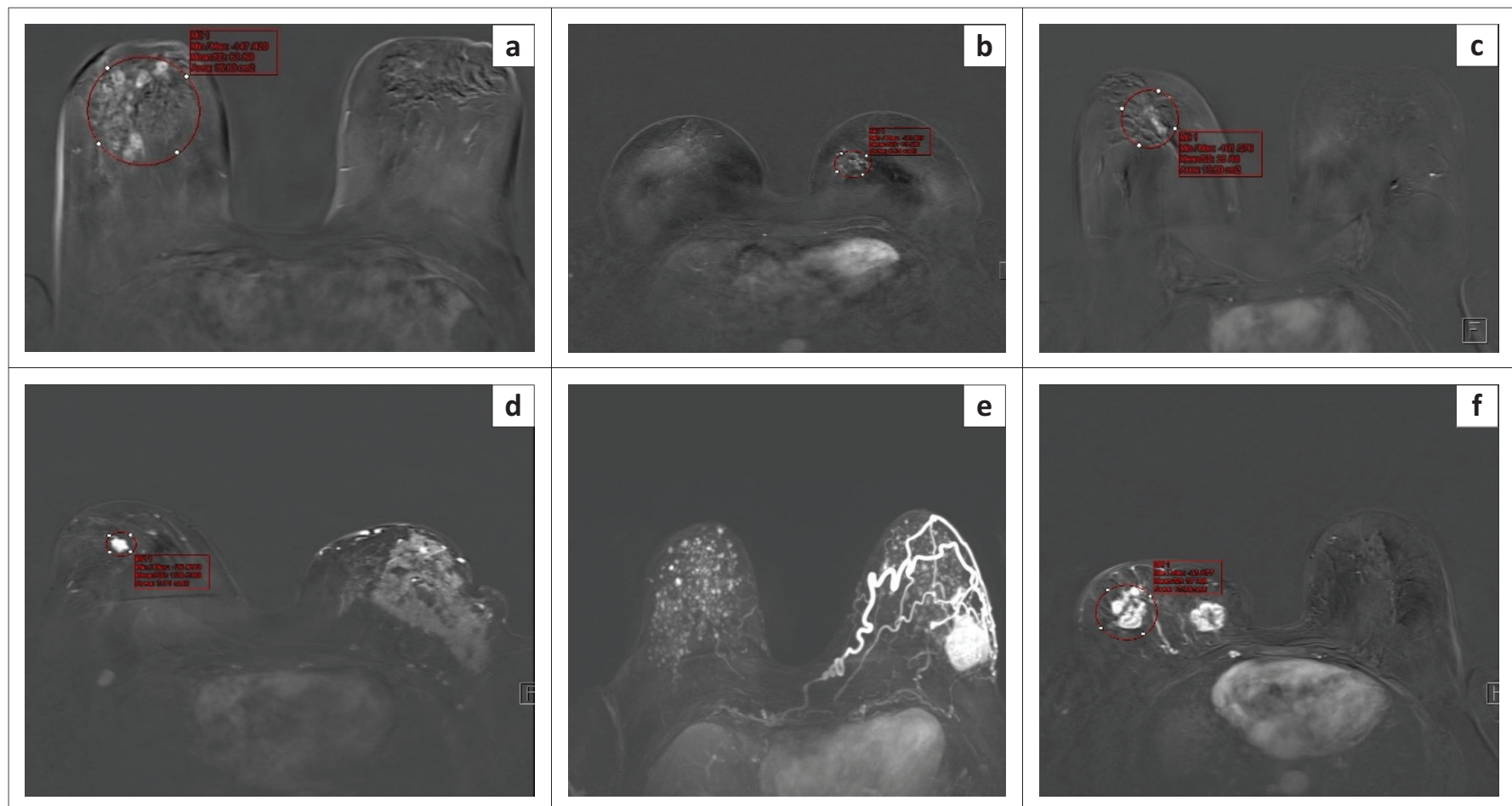

FIGURE 1: Dynamic contrast-enhanced magnetic resonance imaging enhancement characteristic of various patients with breast cancer: (a) clustered ring, (b) clumped, (c) linear distribution, (d) homogeneous (e) larger homogeneous pattern and (f) multicentric heterogeneous enhancement.
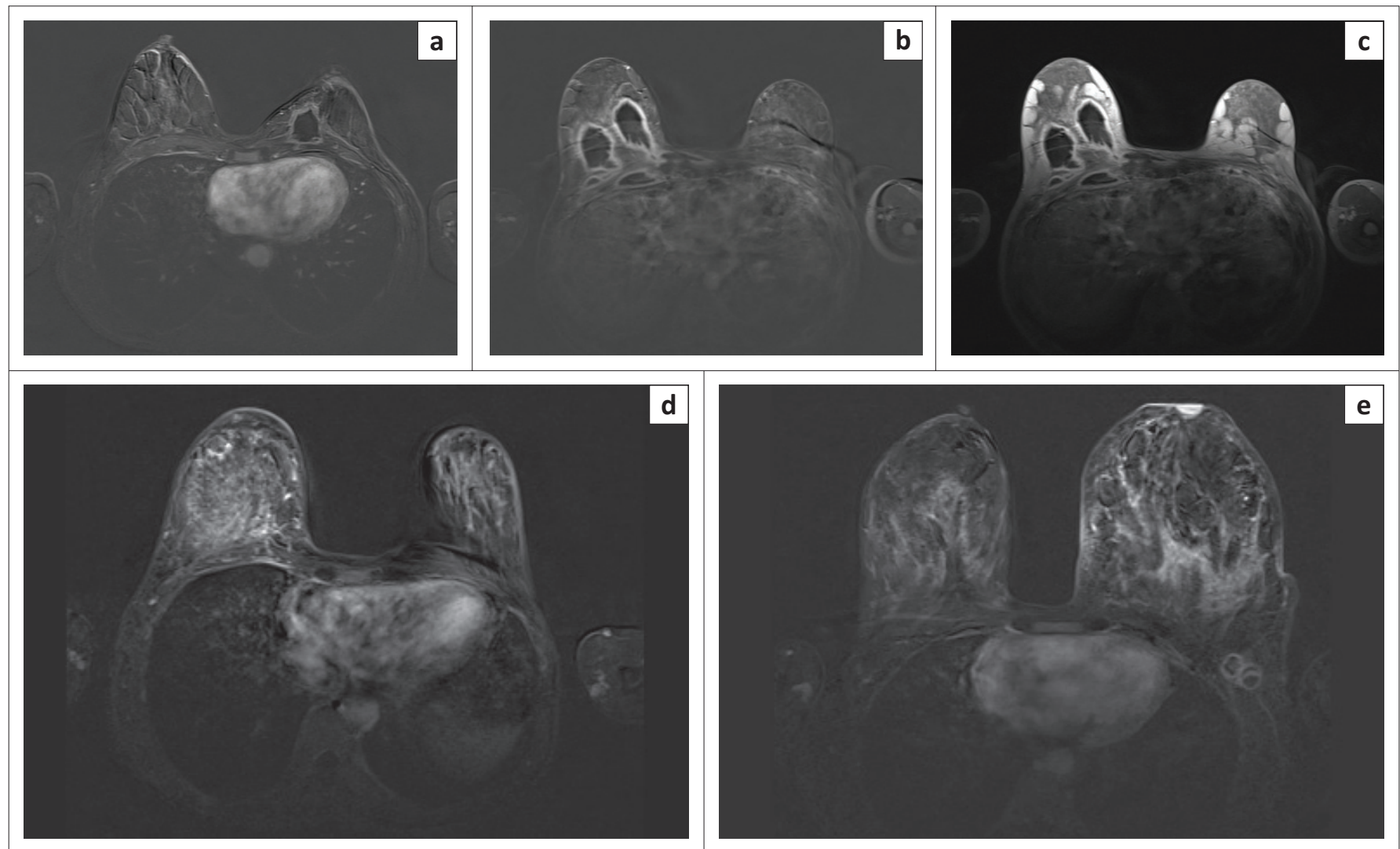

FIGURE 2: Dynamic contrast-enhanced magnetic resonance imaging enhancement patterns of various patients with breast tuberculosis: (a) focal rim, (b) multiple regions rim, (c) same patient as (b) to better demonstrate multiple rim enhancement on the non-subtracted T1-weighted image, (d) clumped diffuse and (e) clumped diffuse associated with rim-enhancing left axillary nodes. 

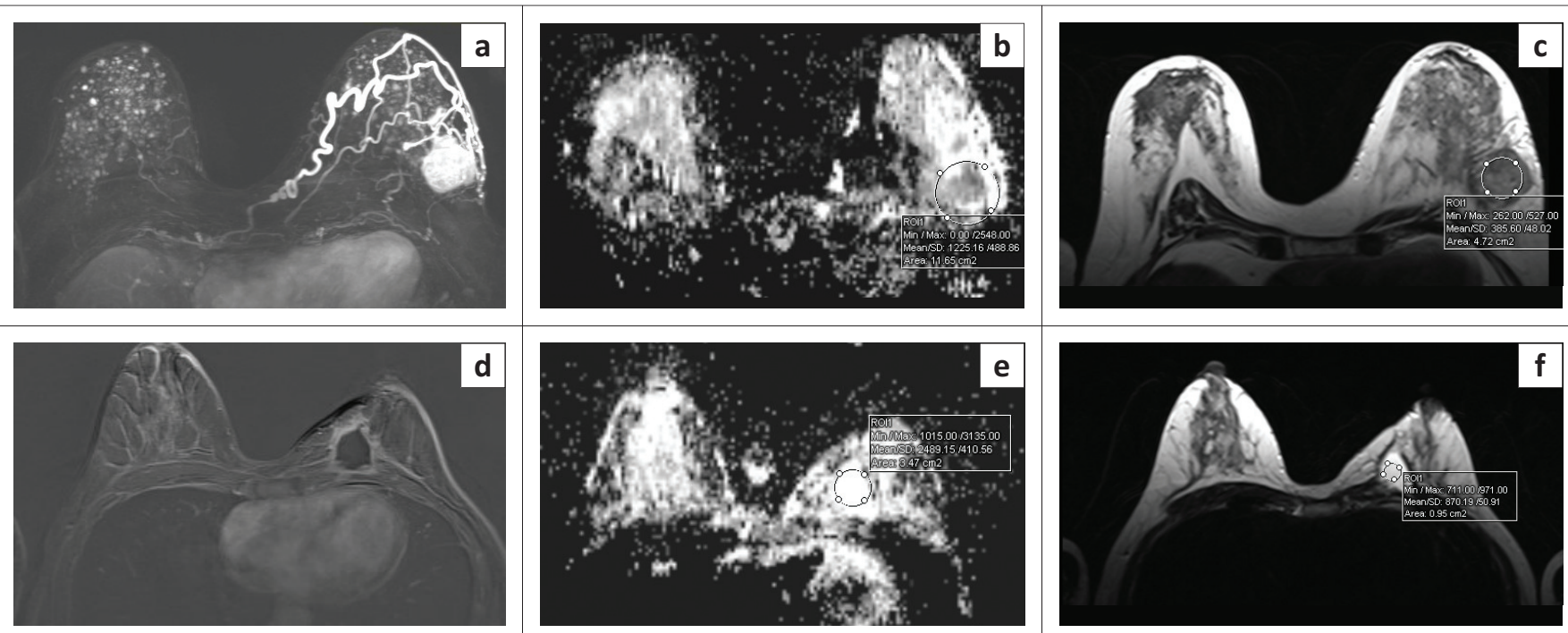

FIGURE 3: Apparent diffusion coefficient value and the T2 signal intensity measurements. (a-c) Axial dynamic contrast-enhanced magnetic resonance imaging (DCE-MRI), diffusion-weighted imaging (DWI) and T2-weighted (T2W)-MRI images of a 61-year-old female patient with left breast cancer: (a) Axial T1 post-contrast subtracted image demonstrates strongly homogeneous enhancing mass with neovascularity in the left breast. (b) Corresponding axial DWI-MRI apparent diffusion coefficient (ADC) map image shows dark signal. (c) Axial T2-weighted image shows the tumour hypointensity. (d-f) Axial DCE-MRI, DWI and T2W-MRI images of a 41-year-old female patient with left breast tuberculosis: (d) Axial T1 post-contrast subtracted image demonstrates a hypointense rim-enhancing mass in left breast; (e) Corresponding axial DWI-MRI ADC map image demonstrates a bright signal; and (f) Axial T2-weighted image shows a uniformly hyperintense signal.

TABLE 2: Comparison of the diffusion-weighted imaging and T2-weighted magnetic resonance imaging quantitative parameters for the breast tuberculosis and breast cancer patients.

\begin{tabular}{|c|c|c|c|c|c|}
\hline \multirow[t]{2}{*}{ Parameter } & \multicolumn{2}{|c|}{ BTB $(n=6)$} & \multicolumn{2}{|c|}{ BCA $(n=17)$} & \multirow[t]{2}{*}{$\bar{p}$} \\
\hline & Mean \pm SD & $95 \% \mathrm{Cl}$ & Mean \pm SD & $95 \% \mathrm{Cl}$ & \\
\hline $\mathrm{ADC}\left(\times 10^{-6} \mathrm{~mm}^{2} / \mathrm{s}\right)$ & $1690.8 \pm 624.1$ & $1035.9-2345.7$ & $1066.3 \pm 375.5$ & $873.2-1259.4$ & 0.008 \\
\hline
\end{tabular}

$\mathrm{ADC}$, apparent diffusion coefficient; BCA, breast cancer; BTB, breast tuberculosis; T2SI, T2 signal intensity value.

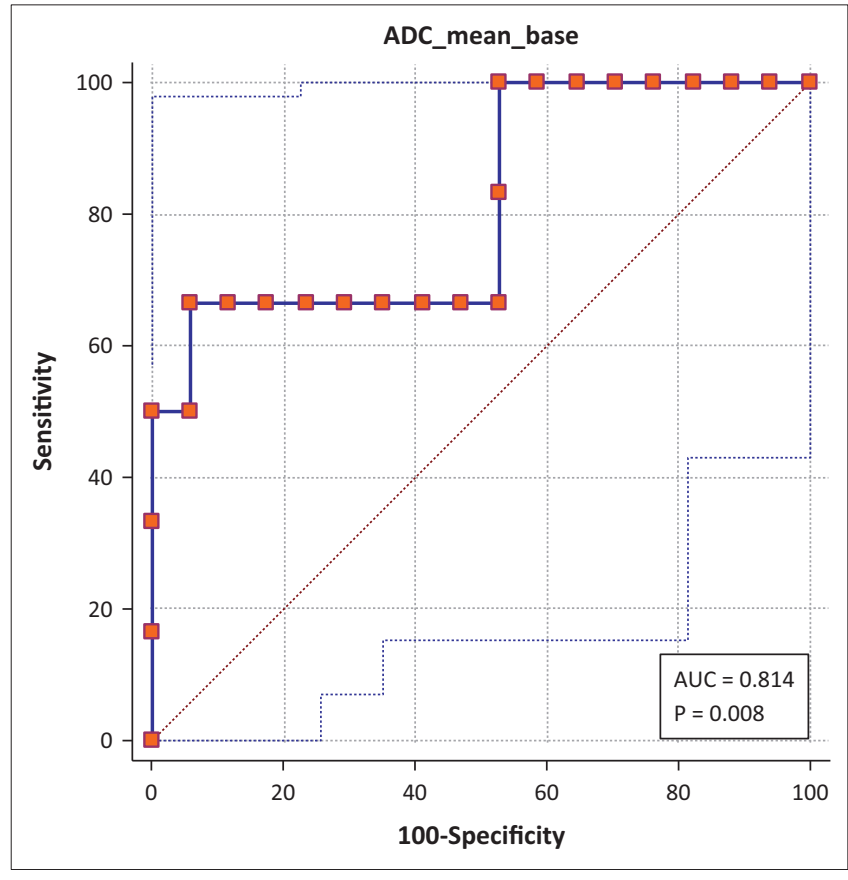

FIGURE 4: Preliminary receiver operating characteristic curve analysis of the apparent diffusion coefficient value of the breast lesions for differentiating cancer from tuberculosis.

and non-mass enhancement (NME) and compared with ANOVA. Significance was shown for both mean ADC value $(p=0.040)$ and T2SI $(p=0.015)$.

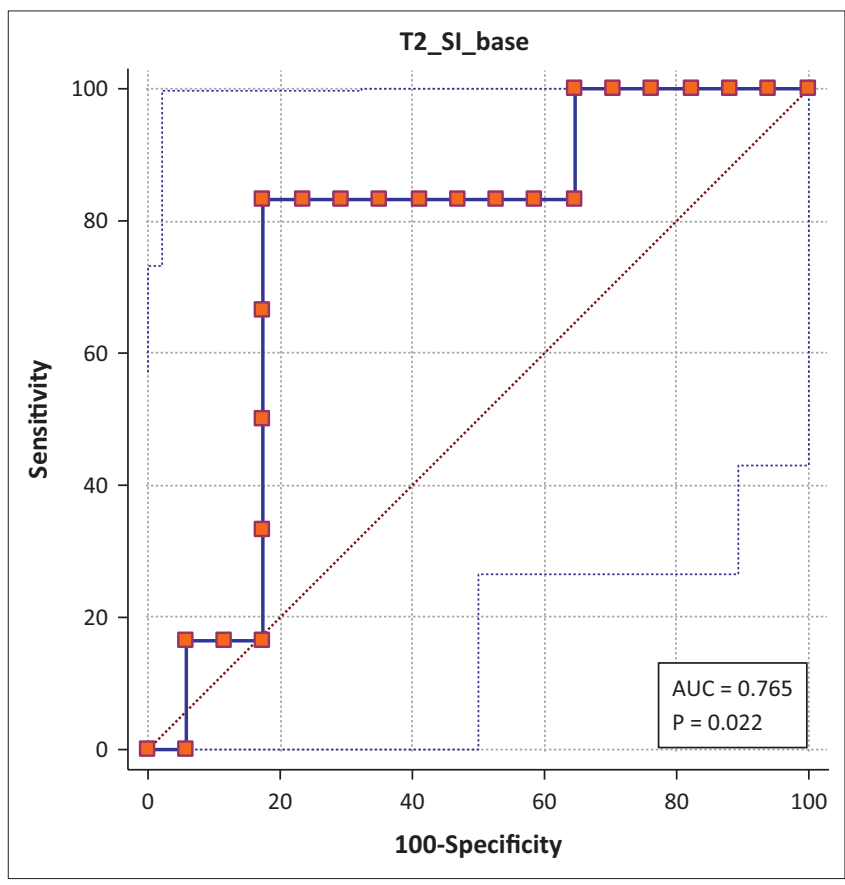

FIGURE 5: Preliminary receiver operating characteristic curve analysis of the T2 signal intensity of the breast lesions for differentiating cancer from tuberculosis.

\section{Discussion}

This study evaluated the ability of the two quantitative MRI markers, the ADC value and the T2SI, and the qualitative 
dynamic post-contrast enhancement patterns to discriminate between BCA and BTB.

\section{Utility}

We have shown that BCA patients have significantly lower mean ADC and T2SI values compared to BTB patients. ROC curve analysis suggests that the ADC value is a better discriminator between $\mathrm{BCA}$ and $\mathrm{BTB}$; however, the confidence intervals are unacceptably wide and further validation, using a larger sample, is required before these can be accepted as having real diagnostic value.

\section{Explanation}

This outcome is explicable in that malignant lesions have high cellularity with resulting restricted diffusion, yielding lower ADC values, a parameter derived from the DWI. This finding has been shown to be consistent across many studies, ${ }^{23,24,25,26,27,38,39}$ with few exceptions. ${ }^{12}$ Woodhams et al. ${ }^{24}$ found malignant breast tumours to have lower ADC values than benign lesions; furthermore, the IDC displayed lower values when compared to the non-invasive ductal cancer (NIDC). In contrast, Chatterjee et al. ${ }^{12}$ found similar ADC values for brain tuberculomas and brain metastases.

The studies that utilised T2SI to discriminate the benign from the malignant breast lesions predominantly focused on the qualitative T2 signal morphologic appearance rather than the quantitative value as in our study. ${ }^{34,35}$ The data on the use of quantitative T2SI as a discriminator between benign and malignant breast lesions are scanty. ${ }^{40}$ Other studies in which T2SI quantitative values were used in differentiating benign from malignant lesions involve other parts of the body and not the breast. ${ }^{41,42}$ Henz et al. ${ }^{41}$ investigated pulmonary nodules with MRI using the quantitative parameters ADC and T2SI in a granulomatous endemic area. They found both the mean T2SI ratio and the ADC to be significant in differentiating the benign from the malignant pulmonary nodules.

Post-contrast morphological analysis revealed that the homogeneous internal enhancement pattern was exclusively observed in BCA patients. This qualitative feature can be used as a further descriptor for separating the two conditions when other imaging or pathology results are equivocal. The diffuse distribution of BTB was visually associated with enlargement of the breast; although this appearance can be confused with inflammatory BCA, the presence of rim-enhancing axillary nodes is more supportive of $\mathrm{BTB}$ than BCA. ${ }^{43}$

\section{Conclusion}

Based on our findings, we conclude that the combination of DCE-MRI morphologic enhancement pattern, the T2SI and the quantitative DWI ADC values may provide some useful non-invasive information in distinguishing malignant from non-malignant illness (in this case, BTB) in patients with suspicious breast lesions. Development of accurate diagnostic algorithms will require accumulation of a larger patient database for BTB in particular; more broadly, however, continued investigation is appropriate in terms of the development of tests to distinguish malignant from benign disease more generally. As with mammographic and ultrasonic findings, the MRI findings alone are not sufficient to distinguish BTB and BCA with acceptable accuracy, and histology remains essential for this purpose.

\section{Acknowledgements}

This publication was made possible by grant number: R24TW008863 from the Office of the US Global AIDS Coordinator and the US Department of Health and Human Services, National Institutes of Health (NIH OAR and NIH ORWH). Its contents are solely the responsibility of the authors and do not necessarily represent the official views of the government. Further funding for operational costs was obtained from the University of KwaZulu-Natal (UKZN) strategic funds. The authors also wish to acknowledge the Medical Education Partnership Initiative (MEPI) for providing seed funding.

\section{Competing interests}

The author declares that they have no financial or personal relationships that may have inappropriately influenced them in writing this article.

\section{Authors' contribution}

D.P.R. contributed to the preparation of protocol and recruitment of participants, including personally obtaining signed consent forms, supervision of radiology investigations, interpretation and processing of images, and drafting of the manuscript for publication. R.J.H. conceptualised the project and contributed to protocol preparation. He further contributed to the editing of the manuscript.

\section{References}

1. Cancer Association of South Africa. Fact sheet on the top ten cancers per population group. CANSA; 2010.

2. Shushtari MHS, Alavi SM, Talaeizadeh A. Breast tuberculosis: Report of nine cases of extra pulmonary tuberculosis with breast mass. Pak J Med Sci. 2011;27(3):582-585.

3. Ramaema DP, Buccimazza I, Hift RJ. Prevalence of breast tuberculosis: Retrospective analysis of 65 patients attending a tertiary hospital in Durban, South Africa. S Afr Med J. 2015;105(10):866-869.

4. Akbulut S, Sogutcu N, Yagmur Y. Coexistence of breast cancer and tuberculosis in axillary lymph nodes: A case report and literature review. Breast Cancer Res Treat. 2011;130(3):1037-1042. https://doi.org/10.1007/s10549-011-1634-8

5. Yang $Z$, Kong $Y$, Wilson $F$, et al. Identification of risk factors for extrapulmonary tuberculosis. Clin Infect Dis. 2004;38(2):199-205. https://doi.org/10.1007/ s10549-011-1634-8

6. Aurum Institute. Managing TB in a new era of diagnosis [homepage on the Internet]. Johannesburg, South Africa: Aurum Institute; 2013 [cited 2014 Sept 15]. Available from: http://www.sahivsoc.org/upload/documents/Aurum $\% 20$ Managing\%20TB\%20in\%20an\%20era\%20of\%20new\%20diagnostics.pdf

7. Leeds IL, Magee MJ, Kurbatova EV, et al. Site of extrapulmonary tuberculosis is associated with HIV infection. Clin Infect Dis. 2012;55(1):75-81. https://doi. org/10.1093/cid/cis303

8. Department of Health (South Africa). National tuberculosis management guidelines 2014 [homepage on the Internet]. Pretoria: Department of Health;
2014 [cited 2014 Nov 20]. Available from: http://www.hst.org.za/sites/default/ 2014 [cited 2014 Nov 20]. Available from: http:/
files/NTCP_Adult_TB-Guidelines-27.5.2014.pdf 
9. Gill M, Chabbra S, Sangwan M, Singh S, Praveen, Tanwar P. Tuberculous mastitis - A great mimicker. Asian Pac J Trop Dis. 2012;2(5):348-351. https://doi.org/10.1016/ S2222-1808(12)60076-0

10. Kapan M, Toksöz Mt, Baklr ŞD, et al. Tuberculosis of breast. Eur J Gen Med. 2010;7(2):216-219. https://doi.org/10.29333/ejgm/82856

11. Baharoon S. Tuberculosis of the breast. Ann Thorac Med. 2008;3(3):110-114 https://doi.org/10.4103/1817-1737.41918

12. Chatterjee S, Saini J, Kesavadas C, Arvinda HR, Jolappara M, Gupta AK Differentiation of tubercular infection and metastasis presenting as ring enhancing lesion by diffusion and perfusion magnetic resonance imaging. J Neuroradiol. 2010;37(3):167-171. https://doi.org/10.1016/j.neurad.2009.08.005

13. Peng J, Ouyang $Y$, Fang WD, et al. Differentiation of intracranial tuberculomas and high grade gliomas using proton MR spectroscopy and diffusion MR imaging. Eur J Radiol. 2012;81(12):4057-4063. https://doi.org/10.1016/j.ejrad.2012.06.005

14. Sankhe S, Baheti A, Ihare A, Mathur S, Dabhade P, Sarode A. Perfusion magnetic resonance imaging characteristics of intracerebral tuberculomas and its role in differentiating tuberculomas from metastases. Acta Radiol. 2013;54(3):307-312. https://doi.org/10.1258/ar.2012.120347

15. Lang N, Su MY, Yu HJ, Yuan H. Differentiation of tuberculosis and metastatic cancer in the spine using dynamic contrast-enhanced MRI. Eur Spine J. 2015;24(8):17291737. https://doi.org/10.1007/s00586-015-3851-z

16. Pui MH, Mitha A, Rae WI, Corr P. Diffusion-weighted magnetic resonance imaging of spinal infection and malignancy. J Neuroimaging. 2005;15(2):164-170. https:// doi.org/10.1111/j.1552-6569.2005.tb00302.x

17. Chung $M H$, Lee HG, Kwon SS, Park SH. MR imaging of solitary pulmonary lesion: Emphasis on tuberculomas and comparison with tumors. J Magn Reson Imaging. 2000;11(6):629-637. https://doi.org/10.1002/1522-2586(200006) Imaging. 2000;11(6):629-637. https://d
11:6\%3C629::AID-JMRI9\%3E3.0.CO;2-R

18. Donmez FY, Yekeler E, Saeidi V, Tunaci A, Tunaci M, Acunas G. Dynamic contrast enhancement patterns of solitary pulmonary nodules on $3 \mathrm{D}$ gradient-recalled echo MRI. Am J Roentgenol. 2007;189(6):1380-1386. https://doi.org/10.2214/ echo MRI. A
AJR.07.2429

19. Peng G, Cai Z, Gao Y. The value of CT and MRI in differentiating malignant nodule from tuberculoma. Zhonghua Jie He He Hu Xi Za Zhi. 1995;18(4):218-220, 255.

20. Schaefer JF, Vollmar J, Wiskirchen J, et al. Differentiation between malignant and benign solitary pulmonary nodules with proton density weighted and ECG-gated magnetic resonance imaging. Eur J Med Res. 2006;11(12):527-533.

21. Popli MB, Kumari A, Popli V. Proton magnetic resonance spectroscopy in breast tuberculosis. Eur J Radiol Extra. 2010;74(3):e59-e63. https://doi.org/10.1016/j. ejrex.2010.04.005

22. Dorrius MD, Dijkstra H, Oudkerk M, Sijens PE. Effect of $b$ value and pre-admission of contrast on diagnostic accuracy of 1.5-T breast DWI: A systematic review and meta-analysis. Eur Radiol. 2014;24(11):2835-2847. https://doi.org/10.1007/ s00330-014-3338-z

23. Qu RF, Guo DR, Chang ZX, et al. Differential diagnosis of benign and malignan breast tumors using apparent diffusion coefficient value measured through diffusion-weighted magnetic resonance imaging. J Comput Assist Tomogr. 2015;39(4):513-522. https://doi.org/10.1097/RCT.0000000000000226

24. Woodhams R, Matsunaga $K$, Kan $S$, et al. ADC mapping of benign and malignant breast tumors. Magn Reson Med Sci. 2005;4(1):35-42. https://doi.org/10.2463/ mrms.4.35

25. Yamaguchi K, Schacht D, Nakazono T, Irie H, Abe H. Diffusion weighted images of metastatic as compared with nonmetastatic axillary lymph nodes in patients with newly diagnosed breast cancer. J Magn Reson Imaging. 2015;42(3):771-778. https://doi.org/10.1002/jmri.24829
26. Yili Z, Xiaoyan $\mathrm{H}$, Hongwen $\mathrm{D}$, et al. The value of diffusion-weighted imaging in assessing the ADC changes of tissues adjacent to breast carcinoma. BMC Cancer. 2009;9:18. https://doi.org/10.1186/1471-2407-9-18

27. Zhao J, Guan H, Li M, Gu H, Qin J, Wu X. Significance of the ADC ratio in the differential diagnosis of breast lesions. Acta Radiol. 2016;57(4):422-429.

28. Thomassin-Naggara I, De Bazelaire C, Chopier J, Bazot M, Marsault C, Trop I. Diffusion-weighted MR imaging of the breast: Advantages and pitfalls. Eur Radiol. 2013;82(3):435-443. https://doi.org/10.1186/1471-2407-9-18

29. Petralia G, Bonello L, Priolo F, Summers P, Bellomi M. Breast MR with special focus on DW-MRI and DCE-MRI. Cancer Imaging. 2011;11:76-90. https://doi org/10.1102/1470-7330.2011.0014

30. Tozaki M. Interpretation of breast MRI: Correlation of kinetic and morphological parameters with pathological findings. Mag Reson Med Sci. 2004;3(4):189-197. https://doi.org/10.2463/mrms.3.189

31. Hnilicova P, Jaunky T, Baranovicova E, Heckova E, Dobrota D. A new approach in DCE MRI data analysis for differentiating benign and malignant breast lesions. Klin Onkol. 2015;28(1):44-50. https://doi.org/10.14735/amko201544

32. Padhani AR. Dynamic contrast-enhanced MRI in clinical oncology: Current status and future directions. J Magn Reson Imaging. 2002;16(4):407-422. https://doi. org/10.1002/jmri.10176

33. Nelson KL, Runge VM. Basic principles of MR contrast. Top Magn Reson Imaging. 1995;7(3):124-136. https://doi.org/10.1097/00002142-199500730-00002

34. Huang YH, Chang YC, Huang CS, Wu TJ, Chen JH, Chang RF. Computer-aided diagnosis of mass-like lesion in breast MRI: Differential analysis of the 3-D morphology between benign and malignant tumors. Comput Methods Programs Biomed. 2013;112(3):508-517. https://doi.org/10.1016/j.cmpb.2013.08.016

35. Tan SL, Rahmat K, Rozalli Fl, et al. Differentiation between benign and malignant breast lesions using quantitative diffusion-weighted sequence on 3 T MRI. Clin Radiol. 2014;69(1):63-71. https://doi.org/10.1016/j.crad.2013.08.007

36. Malich A, Fischer DR, Wurdinger S, et al. Potential MRI interpretation model: Differentiation of benign from malignant breast masses. Am J Roentgenol. 2005;185(4):964-970. https://doi.org/10.2214/AJR.04.1073

37. D'Orsi C, Sickles E, Mendelson E, Morris E. ACR BI-RADS Atlas: Breast imaging reporting and data system. Reston, VA: American College of Radiology; 2013.

38. Imamura $T$, Isomoto I, Sueyoshi $E$, et al. Diagnostic performance of ADC for Non-mass-like breast lesions on MR imaging. Magn Reson Med Sci. 2010;9(4):217-225. https://doi.org/10.2214/AJR.04.1073

39. Yabuuchi $H$, Matsuo $Y$, Kamitani T, et al. Non-mass-like enhancement on contrastenhanced breast MR imaging: Lesion characterization using combination of dynamic contrast-enhanced and diffusion-weighted MR images. Eur I Radiol. 2010;75(1):e126-e132. https://doi.org/10.1016/j.ejrad.2009.09.013

40. Li C, Meng S, Yang X, Wang J, Hu J. The value of T2* in differentiating metastatic from benign axillary lymph nodes in patients with breast cancer - A preliminary in vivo study. PLoS One. 2014;9(1):e84038. https://doi.org/10.1371/journal. pone.0084038

41. Henz Concatto N, Watte G, Marchiori E, et al. Magnetic resonance imaging of pulmonary nodules: Accuracy in a granulomatous disease-endemic region. Eur Radiol. 2016;26(9):2915-20. https://doi.org/10.1007/s00330-015-4125-1

42. Peng $Y$, Jiang $Y$, Antic T, Giger ML, Eggener SE, Oto $A$. Validation of quantitative analysis of multiparametric prostate MR images for prostate cancer detection and aggressiveness assessment: A cross-imager study. Radiology. 2014;271(2): 461-471. https://doi.org/10.1148/radiol.14131320

43. Sathekge AMM, D’Asseler Y, Vorster M, Gongxeka H, Van de Wiele C. Tuberculous lymphadenitis: FDG PET and CT findings in responsive and nonresponsive disease. Eur J Nucl Med Mol Imaging 2012;39(7):7. 